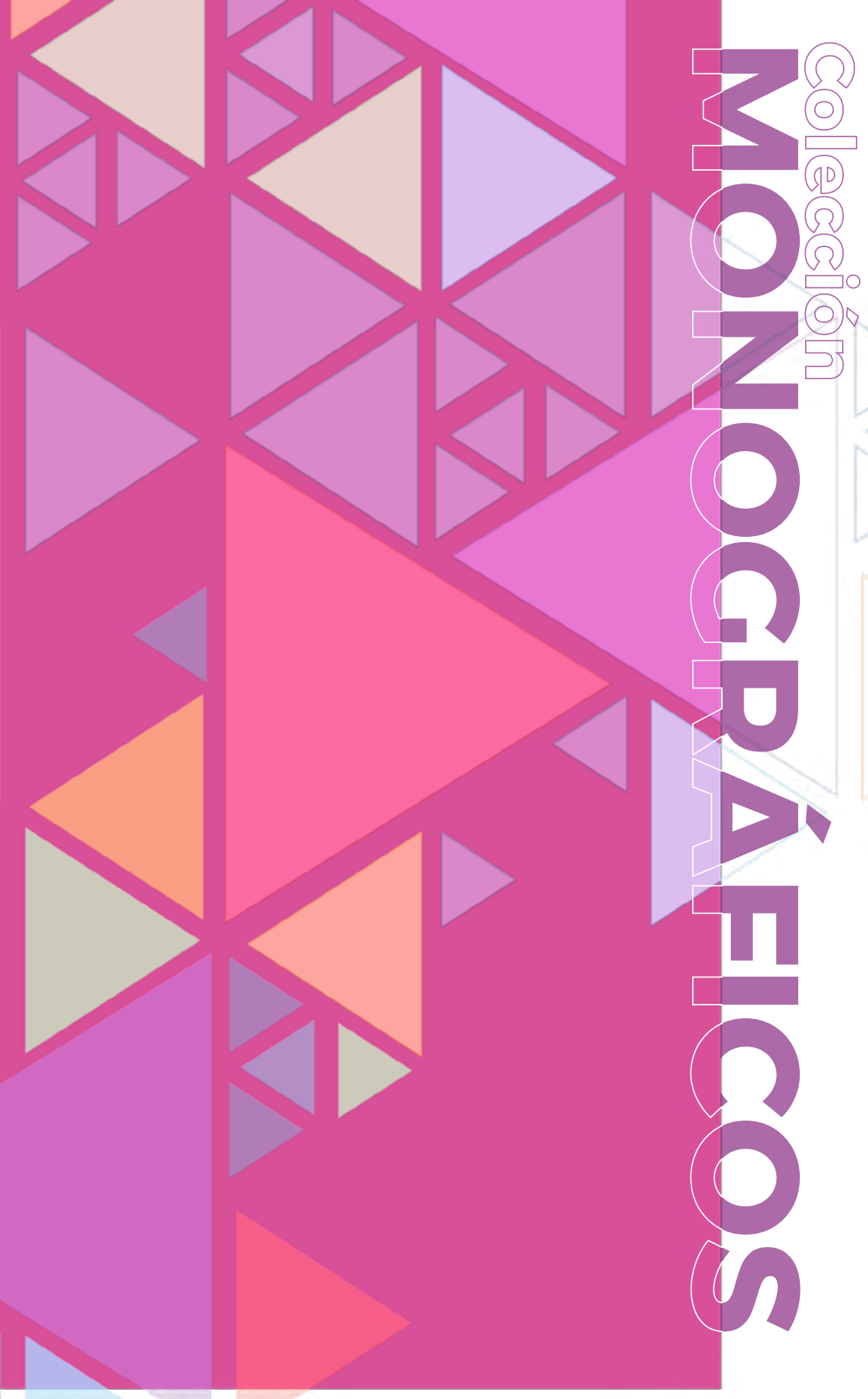

Responsabilidad social empresarial:

Prácticas y perspectivas de la gestión organizacional en el contexto ecuatoriano

Mariana Del Rocío Verdezoto Reinoso Compiladora

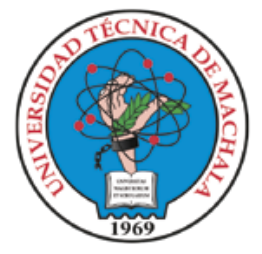


Ediciones UTMACH

147 pág: $21 \times 29,7 \mathrm{~cm}$

Colección Monográficos

Título: Responsabilidad social empresarial: prácticas y perspectivas de la gestión organizacional en el contexto ecuatoriano - Mariana

Del Rocío Verdezoto Reinoso (Compiladora)

Primera edición 2020

ISBN: 978-9942-24-140-5

CDD 338,9

1. Desarrollo y crecimeinto económico,

2. Empresa y gestión

--Temas relacionados

Publicación PDF 
Responsabilidad social empresarial: prácticas y perspectivas de la gestión organizacional en el contexto ecuatoriano

Mariana Del Rocío Verdezoto Reinoso

COMPILADORA

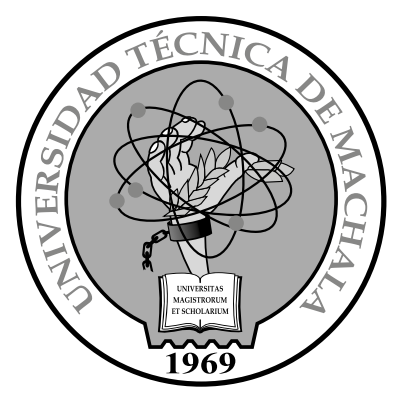


Autoridades

César Quezada Abad - Rector Amarilis Borja Herrera - Vicerrector Académico Jhonny Pérez Rodríguez - Vicerrector Administrativo

Luis Brito Gaona

Director de Investigación

(c) Ediciones UTMACH Colección Monográficos

Título original:

Responsabilidad social empresarial: prácticas y perspectivas de la gestión organizacional en el contexto ecuatoriano

ISBN: 978-9942-24-140-5

(c) Mariana Del Rocío Verdezoto Reinoso

(Compiladora)

(c) Autores de capítulos

DOI: http://doi.org/10.48190/9789942241405

Primera edición 2020

Karina Lozano Zambrano Jefe editor / Diseño y edición editorial

Fernanda Tusa Jumbo - Corrector de estilos Jorge Maza-Cordova - Asesor tecnológico

Karla Ibañez y Cyndi Aguilar - Equipo de difusión

Este obra está bajo una licencia de Creative Commons Reconocimiento No Comercial.

Esta licencia permite a otros entremezclar, ajustar y construir a partir de su obra con fines no comerciales, siempre y cuando le reconozcan la autoría y sus nuevas creaciones estén bajo una licencia con los mismos términos. 


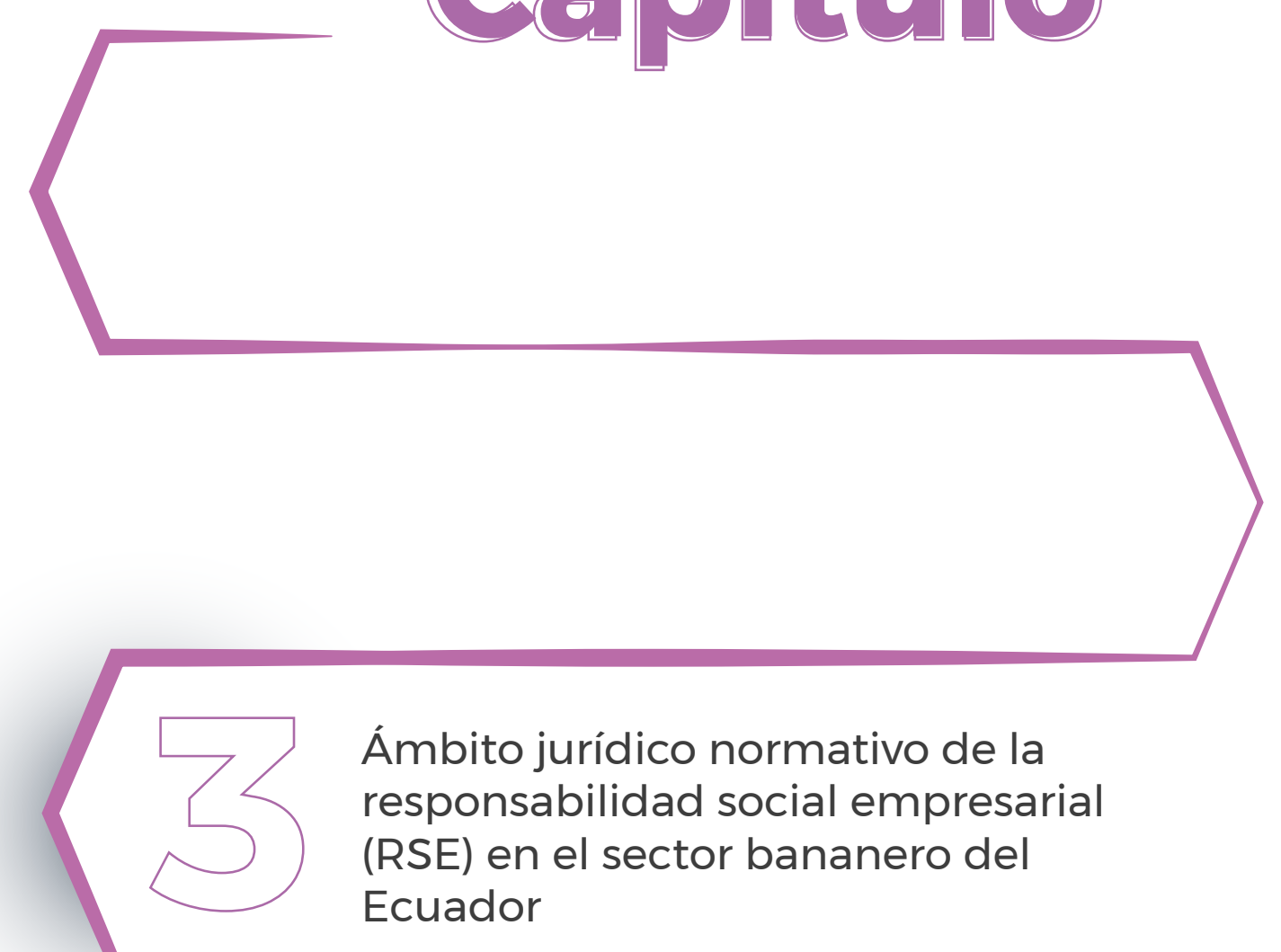


Ámbito jurídico normativo de la responsabilidad social empresarial (RSE) en el sector bananero del Ecuador 


\section{Ámbito jurídico normativo de la responsabilidad social empresarial (RSE) en el sector bananero del Ecuador}

\section{Rocío de Lourdes Guzmán Arias}

Docente e investigadora de la Universidad Técnica de Machala, Abogada de los Tribunales y Licenciada en Ciencias Políticas y Sociales, Magíster en Derecho Civil, Doctoranda en Derecho- Universidad Da Coruña. 


\section{Resumen}

La importancia de la Responsabilidad Social Empresarial, en adelante RSE, ha evolucionado en los últimos tiempos; en este sentido, el gobierno ecuatoriano ha creado políticas y programas para hacer efectiva su aplicación. En la presente investigación, se analiza la importancia y papel que desempeña el derecho en la aplicación de la RSE en el ordenamiento jurídico ecuatoriano, y cómo estos inciden específicamente en el sector bananero. La normativa jurídica existente al respecto, contempla leyes, reglamentos, decretos, resoluciones, entre otros. Sin embargo, la balanza se inclina al aspecto económico y la protección de los bienes, llamados derechos colectivos o derechos humanos. El objetivo de este trabajo, es analizar la incidencia del marco normativo jurídico respecto a los principios de la RSE, en las áreas de los derechos humanos, ambientales y laborales del sector bananero del Ecuador. En la metodología cuenta con una investigación de tipo documental que abarcó la revisión de trabajos investigativos y normativas, mediante un estudio descriptivo y de corte transversal, basado en información cualitativa. Se concluye que, las normativas de RSE se encuentran vinculadas con las normativas ecuatorianas, así como, en los tratados y convenios Internacionales de que Ecuador es parte y por ende vinculantes, por ejemplo, la Declaración de Derechos Humanos, Convenio Marco de las Naciones Unidas sobre cambio climático, convenio sobre abolición trabajo forzoso, Convenio sobre la discriminación, sobre todo a lo relacionado al respeto de los derechos humanos, al medio ambiente y los derechos laborales de los trabajadores, aspectos relacionados al sector bananero del Ecuador.

Palabras claves: responsabilidad social empresarial, ordenamiento jurídico, sector bananero.

\section{Introducción}

\section{Antecedente}

El mandato constitucional que existe en Ecuador en sus diversas aristas, acompañada con el "Plan toda una vida", nos obliga a plantearnos la interrogante, de que, ¿si en el sector bananero ecuatoriano se cumple con los principios de la RSE? La Constitución ecuatoriana impulsa el régimen del desarrollo mediante sus principios generales que establece directrices para construir un sistema económico sostenible justo y respetuoso de la naturaleza (Morán, 2016). De la lectura rápida que realizamos a la normativa ecuatoriana, en cuanto al cumplimiento de la responsabilidad social empresarial, podría decirse, que si se legisla en cuanto al tema, del análisis hecho a las normativas existentes sobre la RSE, se encontró que se acopla en beneficio de los cuatro aspectos, que son; económicos, laborales, sociales y ambientales. 
El tema de la responsabilidad social empresarial, en los últimos años, ha tenido su auge en todo el mundo, las empresas hacen sus esfuerzos por dar cumplimiento a esta normativa, sin embargo, pese al esfuerzo realizado, se puede evidenciar la vulneración de los derechos humanos y de los derechos de la naturaleza. En ese mismo orden de ideas, la actual constitución del Ecuador en su artículo 71 considera a la naturaleza como sujeto de derechos, y no, objeto de derechos, como era considerada anteriormente (Constitución del Ecuador, 2008). Por lo tanto, surge la necesidad de adaptar la actuación de las empresas, en respeto al medio ambiente.

En Ecuador, se han realizado importantes cambios en cuestión de legislación y exigencias a las empresas, haciendo que las empresas se preocupen cada vez más por la preservación ambiental, concientización de la naturaleza, reducir la desigualdad social. En la actualidad, se toma muchas medidas proteccionistas para el medio ambiente por lo que, las empresas deben acogerse a los lineamientos establecidos para su conservación y que es una responsabilidad y obligación para las empresas (Poveda \& Parrales, 2018). Las organizaciones públicas y privadas para el debido cumplimiento de la RSE, toman en cuenta el marco jurídico vigente, haciendo que sus prácticas empresariales sean responsables.

La RSE llega a generar bienestar y desarrollo en las empresas, así como también en el entorno en donde se desarrolla. Su implementación, especialmente si se realiza de forma adecuada contribuye a que las administraciones empresariales cumplan sus objetivos y generen confianza en su entorno. De esta manera, a partir de la aplicación de estrategias empresariales, las empresas buscan obtener beneficios tanto internos como externos, los mismos que deben verse reflejados en el entorno empresarial y en el medio ambiente, a través del progreso y la mejora en la calidad de las relaciones sociales de la empresa con el entorno en donde se desenvuelve.

En el tema que nos compete, las empresas bananeras en el afán de obtener mayores beneficios, no deben descuidar la ética en sus actuaciones; este tema reviste gran relevancia, debido a que la ética es parte fundamental de todos los aspectos de la vida humana, lo que incluye a las organizaciones, independientemente de la actividad económica a la que se dediquen; y, una de las formas en que se materializa la ética es con el cumplimiento de las leyes establecidas para el efecto.

El irrespeto a los derechos de los colaboradores y por el medio ambiente ha hecho que la RSE alcance un mayor desarrollo en los últimos años, Ecuador ha incluido en sus distintas leyes del país, normativas que indirectamente contribuyen a la responsabilidad social empresarial. Las empresas en su afán de conseguir un desarrollo económico más elevado, realizan prácticas socialmente responsables (Coba, Díaz, Zurita, \& Proaño, 2017). No solo las empresas han tenido la necesidad de aplicar dicha responsabilidad por las buenas prácticas, sino también por proyectar una buena imagen, y también está considerada como parte importante de la sustentabilidad de las empresas, por ello, debería estar incluida en toda cultura organizacional para lograr así un respeto hacia las personas, comunidades y el medio ambiente en general. Por otro lado, algunas empresas, toman a dicha res- 
ponsabilidad como una estrategia para dar una buena imagen; en fin, es analizada desde distintos puntos de vista. En Ecuador, actualmente la responsabilidad social es considerada como un método de sostenibilidad, mejora de procesos y optimización de los recursos que influyen en la reducción de costos (Acosta, 2018).

La RSE solicita de las empresas una buena actuación a nivel corporativo, que se evidencie tanto en el interior como en el exterior de la misma. Esto significa que son necesarias establecer buenas relaciones con el personal que labora en las bananeras del país, transparencia en la ejecución de sus actividades, respeto hacia los clientes y preocuparse por el impacto de sus operaciones en el medio ambiente (Wojtarowski, Silva, Piñar, \& Negrete, 2016). Este aspecto es de suma importancia, puesto que al ser Ecuador uno de los países con mayor producción de banano; las bananeras se encuentran muy cerca de zonas pobladas, que, en la mayoría de los casos, se sienten afectadas, por los olores químicos de las fumigaciones aéreas. Frente a estas situaciones el gobierno nacional y local han impulsado la adopción de políticas formales para difundir la RSE, motivando en el hecho de que puede favorecer en la convivencia armónica, así como, con las ventajas competitivas en determinados mercados que buscan entablar relaciones con empresas socialmente responsables (Chumaceiro, Hernández, \& Ziritt, 2013).

En otro orden de ideas, las empresas medianas y pequeñas - Pymes, llevan a cabo prácticas de RSE de forma incipiente, y, sus esfuerzos se traducen en acciones poco sistemáticas. En Ecuador se han llevado a cabo grandes esfuerzos para que las empresas cumplan con las leyes ambientales y realicen las inversiones necesarias para un correcto funcionamiento, siendo esto un factor determinante para que las empresas consideren importante a la RSE (Coba, Díaz, Zurita, \& Proaño, 2017). Por lo tanto, se estima que en Ecuador existe un avance significativo en prácticas como: producción orgánica y comercio justo, además del impulso a un turismo responsable, enfocados en un mercado que conoce la importancia de este tema (Villacís, Suárez, \& Güillín, 2016).

\section{Justificación}

La presente investigación tiene su impacto, en la medida que el derecho y el ordenamiento jurídico de un país, no es estático, sino al contrario, es dinámico, y, en ese dinamismo obedece a las necesidades que reclama la sociedad, siendo una de esas necesidades, el cuidado de la naturaleza, protección en el ámbito laboral dentro de una empresa, el aspecto social, y por supuesto, el aspecto económico, que es el generador clave en la riqueza de un país.

La Constitución ecuatoriana del 2008, señala que Ecuador es un "Estado de Derecho y Justicia Social” (CE, 2008), por lo cual, el estado debe regir su actuación, al cumplimiento de las responsabilidades sociales en beneficio de la comunidad toda. En este orden de ideas, hablar de responsabilidad social empresarial, se está hablando también de desarrollo sostenible y sustentable, que nos sirve para elevar 
la calidad de vida de todas las personas miembros de un país, sin menoscabar los derechos consagrados a la naturaleza, ni los derechos de las personas trabajadoras; y, al centrarse la investigación en el sector bananero, el cual, es un sector que abarca muchas áreas a proteger, por ejemplo: el medio ambiente, ámbito laboral con el respeto al derecho de los trabajadores, impacto social y apoyo a la comunidad o acción social, sector productivo y económico, pago de impuestos, la igualdad de condiciones, entre otros.

La posición de la autora en este capítulo, es sostener que la RSE constituye un nuevo reto para el Derecho, porque en Ecuador falta aún mucho por hacer en materia legislativa, la RSE no se ha regulado de manera específica, sino más bien, existen un conjunto de normas independientes que merecen ser refundidas en un solo cuerpo normativo, por lo tanto, este capítulo pretende abrir el debate jurídico en torno a esta figura, para proponer cambios sustanciales dentro del abanico del ordenamiento jurídico ecuatoriano, con respecto a la RSE.

\section{Objetivo general}

Analizar la incidencia del marco normativo jurídico respecto a los principios de la RSE en las áreas de los derechos humanos, ambientales y laborales del sector bananero del Ecuador.

\section{Objetivos específicos}

- Revisar la evolución de la normativa nacional e internacional acerca de la RSE.

- Analizar el impacto de la RSE en la actividad del sector bananero del Ecuador, desde el ámbito del ordenamiento jurídico establecido.

\section{Materiales y métodos}

En la presente investigación se utilizó una metodología de análisis - síntesis. Es una investigación de alcance descriptivo y de corte transversal, basada en información cualitativa. Se revisaron diversas normativas sobre la temática, tanto nacionales como internacionales, destacando su crecimiento normativo a nivel mundial. Se hizo una revisión bibliográfica para identificar fuentes primarias relacionadas a la RSE a nivel internacional y su aplicación en Ecuador. 


\section{Resultados y discusión}

Uno de los fines importantes de esta investigación fue tener una visión clara de la perspectiva de la RSE en el sector bananero, teniendo en cuenta las normativas que se han ido desarrollando y que van en armonía con esta temática, sobre todo lo relacionado con el medio ambiente y el derecho de los trabajadores. Es evidente la lucha que tienen que continuar los sectores afectados; en el tema laboral sólo existe un pequeño avance en las Comisiones sectoriales de los trabajadores, carecen de programas de salud ocupacional y ambiental del sector bananero. En el área ambiental, Ecuador acoge algunos tratados y convenios internacionales que coadyuvan a la protección de la naturaleza, tales como, Declaración de Río sobre Medioambiente y Desarrollo, Convenio Marco de las Naciones Unidas sobre cambio climático, el Protocolo de Kioto. Estos tratados y convenios van de la mano con lo establecido en la Carta Magna ecuatoriana con respecto a los derechos de la naturaleza.

Se evidencia una marcada tendencia en varios países, incluido Ecuador, en reglar y normar con respecto a la RSE, sobre todo acogerse a varios acuerdos entre actores globales, los mismos que son tendencias a nivel mundial y van de la mano con los Derechos Humanos, los Principios de la Organización Internacional del Trabajo (OIT), el Desarrollo y la Convención de la Organización de las Naciones Unidas (ONU) contra la corrupción.

El debate sobre la obligatoriedad de la RSE aún se mantiene, sin embargo, los principios especiales de este sistema van cobrando fuerza, a través de las leyes aprobadas de los diferentes convenios y tratados internacionales, los mismos que son vinculantes para Ecuador, como país suscrito. En la legislación ecuatoriana no existe una normativa específica que regule la RSE, algunas leyes mencionan tangencialmente el término, por otra parte, la doctrina realiza ciertas aportaciones, sin que se llegue a un profundo análisis y tratamiento legislativo, por lo tanto, se trataría de un derecho blando. Las iniciativas de RS invitan a las empresas de forma voluntaria a acoger los principios de la RSE, sin que en caso de incumplimiento, se sancione.

\section{La responsabilidad social en el sector bananero}

El sector bananero se caracteriza por ser uno de los sectores productivos más importantes del país, pese a las continuas crisis que le ha sobrevenido, se mantiene en una posición referencial, como generador de divisas en el país y como fuente de trabajo para miles de ecuatorianos. Según (Sánchez, 2016) "La exportación bananera representa también el 2\% del PIB general, 26\% del PIB agrícola, $8 \%$ de las exportaciones generales, $27 \%$ de las exportaciones agropecuarias y $20 \%$ de las exportaciones no petroleras".

La forma como se lleva a cabo el proceso de monocultivo del banano es una de las características más problemáticas del sector, pues el uso de químicos altamente 
peligrosos desencadenan una serie de enfermedades a los trabajadores; enfermedades que van desde enfermedades respiratorias hasta enfermedades catastróficas como el cáncer. Esto debido a la falta de cuidados y políticas de seguridad empleados para el trabajo. Algunas empresas bananeras tienen creadas fundaciones con servicios de salud, sin embargo, el trabajo estas realizan, son muy superficiales, sin prestar mayor atención a las diversas enfermedades existentes, como por ejemplo, trastornos musculares, enfermedades mentales, problemas a los órganos, en la piel, entre otros. Al parecer, algunas empresas crean una especie de cortina de humo, haciendo creer que cumplen con las leyes y fomentan la RSE.

Otra de las problemáticas identificadas en el área laboral dentro del sector bananero es, relacionado a la inexistencia de contratos laborales y pago de salario por debajo de los establecidos en las tablas sectoriales de cada año. Extensión de horarios por más de 12 horas en los tiempos de cosecha y la polivalencia de las tareas del trabajador (Vitali, 2017).También se ha identificado en el sector bananero, la discriminación del trabajo realizado por mujeres, a pesar de que realizan similar trabajo que un hombre, no existe el mismo reconocimiento salarial, a esto se suma, los despidos por situaciones de embarazos, quedando indefensas en un momento muy importante de sus vidas.

Por lo expresado, en Ecuador se han realizados importantes cambios en la legislación; en especial en lo laboral y empresarial. Sin embargo, las empresas de manera general no prestan mucha atención a los temas sociales y ambientales, siendo escasas las empresas que de manera voluntaria implementan la filosofía del RSE de manera sostenible (Coba Molina, Díaz Córdova, Zurita Meza, \& Proaño López, 2017).

\section{Exigencia o voluntariedad en la aplicación de la RSE}

Según lo hemos expresado la RSE y su aplicación en el ámbito empresarial surge de factores voluntarios, sin embargo, en diferentes países del mundo, se han plasmado diferentes normativas en torno a esta temática. Y, Ecuador no se ha quedado rezagado en esta regulación. Cuando nos referimos al carácter voluntario de la aplicación de los instrumentos de la RSE estamos frente al régimen de softlaw, un ejemplo claro de este régimen son los códigos de buen gobierno en donde se expresa la voluntad y la disposición de la empresa para el reconocimiento de todos los intereses en juego (González \& García, 2006).

El softlaw tiene su importancia en la medida que no es exigible para ninguna empresa, y no esta revestido del poder sancionatorio del Estado. Mas bien, se trata de un recurso que es acogido y respetado de manera voluntaria por parte de los involucrados, como parte de un compromiso a respetar. Es decir, es una autorregulación que desean afrontar las empresas. Algunos de los defensores de este sistema, consideran que existe mayor agrado en la satisfacción de los consumidores y clientes y que, por lo tanto, no es necesario que exista la exigibilidad de la ley 
para tener una empresa socialmente responsable (Ibarra Padilla, 2014). Ejemplo de este sistema son los instrumentos internacionales que la RSE quiere establecer de manera voluntaria, quedando a discreción de las empresas el acogerlas, careciendo de fuerza obligatoria y su incumplimiento no genera sanción ni afectación en la empresa(Acevedo Guerrero , Zárate Rueda , \& Garzón Ruiz , 2013).

Lo contrario a este sistema voluntario es el sistema hard law, los defensores de este sistema enfatizan que la RSE no puede dejarse al libre albedrío de las empresas, ya que algunas empresas consideran que la "RSE solo tiene sentido si el costo de desplegar un comportamiento más virtuoso continúa siendo menor que los beneficios” (Vogel, 2005).

Bajo estos criterios, algunos países han optado por incentivar a aquellas empresas que son socialmente responsables, manteniendo una serie de incentivos para aquellas empresas que cumplan con los estándares de RSE, pudiendo ser, rebajas en los impuestos, destacar y aplaudir su gestión públicamente,

La puesta en marcha de la RSE debe ser considerada como un acto voluntario por parte de las empresas, donde impere la consciencia social, mas no, como una imposición al que se deban ajustar. El sector empresarial a más de obtener un beneficio financiero, busca ser bien visto ante la sociedad, porque eso realzará su nivel competitivo, pues, aquellas empresas que son socialmente responsables, son mayormente reconocidas por el consumidor, y esto conlleva a su divulgación, provocando beneficios en la publicidad de la empresa (Lizarzaburu \& Brito , 2016) . Sin embargo, lo que en la actualidad mueve en gran medida al empresario a acogerse a la RSE es tener armonía con la protección a la naturaleza y por ende al entorno social. En este sentido, hoy en día una empresa no puede estar alejada de las exigencias actuales, siendo estas: creatividad, innovación, mejora de la cadena de producción, calidad y sostenibilidad, aceptación de referentes y normativas internacionales, entre otros. En una investigación profunda realizada en Europa, se pudo verificar que por ejemplo, en la mayoría de las empresas europeas no conciben la RS como una nueva forma de hacer negocios, sino como una novedosa herramienta de gestión que les permite realizar acciones responsables y comunicarlas (Sánchez, 2018).

Pues, la alarmante situación que enfrenta el planeta, ha llevado a plantearnos alguna solución para mitigar los daños causados por el hombre. Las consecuencias del calentamiento

se hacen visibles en nuestros días, los desastres naturales se han desencadenado a nivel mundial con efectos desastrosos. La extinción de animales y plantas también nos hacen pensar en una solución. El crecimiento de enfermedades raras y catastróficas también son efectos del mal manejo del medio ambiente. Todos estos ejemplos enunciados son una clara muestra de la urgente necesidad de tener consciencia en la actividad empresarial. Además, tenemos las enfermedades profesionales, y sobre esto, existe la percepción de que las innovaciones no son necesarias, razón por la cual, se siguen utilizando prácticas antiguas que van en desmedro de la salud y condiciones físicas de los trabajadores (Murillo Barrera, 2018). 
Sin embargo, pese a esta difícil situación, podemos también evidenciar que muchos empresarios, llámense pequeños o grandes, no tienen interés en la RSE, sus actuaciones en desmedro de la propiedad privada, inmoralidad, corrupción, afectación a clientes, violación de derechos, entre otros, no tienen límites, lo importante son sus estados financieros (Moya Arana, 2014).

Las organizaciones deben tener claro que es lo que desean con la RSE, si desean ser realmente responsables con la sociedad, y hacer del RSE parte de su estructura organizacional; ser empresas sólo con programas de responsabilidad social, aplicando únicamente uno de los objetivos del RSE; o simplemente negarse a una realidad empresarial que cada vez toma más vigencia en mundo, debido a la globalización(VVAA, 2017).

La aplicación de la RSE en los diferentes sectores no es una tarea tan sencilla, es una cuestión de tiempo. Las empresas deberían contribuir a la formación y capacitación de los jóvenes y futuros empresarios, con programas de divulgación de los conceptos empresariales, de emprendimiento, de buena gobernanza y responsabilidad social, así como de transparencia y ética en los negocios (Olcese, 2015) y de esta forma estamos creando una conciencia de responsabilidad social a los futuros empresarios de la sociedad. También es importante que desde la academia debe reforzarse el tema de adhesión al RSE ya que permite alcanzar sostenibilidad y sustentabilidad (Luzurriaga, 2018).

\section{Evolución de la RSE en el marco normativo internacional}

A partir de los años sesenta, la RSE era conocida bajo el concepto de Corporate Social Responsibility (CSR) en Estados Unidos, luego, esta idea empezó a plasmarse en Europa en el año de 1990. La comisión Europea acoge el concepto CSR haciéndolo oficial en los lineamientos de la Unión Europea, plasmándose en el Libro Verde, documento que en lo posterior servirá de base para que las empresas de manera voluntaria, decidan considerar aspectos económicos, sociales y ecológicos en sus actividades empresariales (Schulze \& Pszolla, 2011).

La comunidad, exige que la actividad empresarial y económica cumpla con un bien común. Es descifrable que las organizaciones busquen como a dé lugar el beneficio propio a un camino de éxito empresarial, siempre y cuando sigan los lineamientos sobre los derechos de una sociedad.

La Responsabilidad Social Empresarial es el conjunto de las acciones innovadoras de una organización, basadas en el cumplimiento de las leyes y en los valores éticos, para institucionalizar un gobierno corporativo transparente que integre en las actividades en curso de la organización la generación de riqueza respetuosa de los derechos humanos con las responsabilidades de sus actividades sobre la sociedad y el medio ambiente. De todas estas acciones debe rendir cuentas a la sociedad. (Martínez, 2010). 
Para concretar la RSE se la puede comprender como una gran cantidad de actividades escrupulosas y metódicas, que las empresas realizan en su interior, para aumentar la satisfacción personal, la protección de la tierra y el avance social de la nación.

Según, (Hurtado, 2008) por lo general, es frecuente encontrar referencias al uso de la Responsabilidad Social en las Empresas en declaraciones públicas o en las comunicaciones generales. Sin embargo, pese a la cantidad de la misma aún existe escases de información académica y científica sobre estos propósitos.

En el siglo XX se empieza a desarrollar la RSE de una manera moderna, Estados Unidos fue uno de los países en donde comenzó a tener un crecimiento económico, lo cual produjo cambios sociales. La separación que se dio entre las propiedades empresariales y la extensión de las mismas organizaciones fueron el principal motivo para impulsar la información y la gestión sobre la responsabilidad social empresarial. (Acuña, 2014).

Otros de los primeros enfoque de la RSE fue la Segunda Guerra Mundial, donde se señaló que las empresas americanas deberían llevar ciertas obligaciones sociales. Se buscada sacar provecho a la búsqueda del interés público por parte de las empresas en apoyar la educación, la estabilidad económica, la solidaridad y conservación de los recursos naturales.

En Ecuador las primeras empresas que empiezan a incorporar este concepto en sus actividades, son las empresas multinacionales y las empresas exportadoras. En relación a esta temática, Ecuador ha tomado impulso, sobre todo a lo relacionado a la producción agrícola, empresas asociativas, empresas turísticas. Sin embargo, esta actuación antes del año 2008, todavía era considerada como aislada, debido a la falta de modelos de gestión con indicadores en materia ambiental y social. La adopción de la RSE como modelo de gestión no es fácil, porque necesitan mantener un equilibrio entre la dimensión social, medioambiental y económica (Armijos, 2017).

Es a partir de la entrada en vigencia de la Constitución del año 2008, en que empieza visualizarse cambios significativos en torno a la RSE. El Plan Nacional del Buen Vivir, también cumplió un papel importante en las políticas de gobierno de ese entonces, donde la frase SumakKawsay era considerada como la meta a seguir, basado en el cumplimiento de responsabilidad social y compromiso ético relacionado con la naturaleza, considerado como pilar básico de la humanidad (Villacís, Suárez, \& Güillín, 2016).

El Instituto de Responsabilidad Social Empresarial, también jugó un papel importante en Ecuador, cuya misión era plasmar una cultura de desarrollo sostenible basada en la RSE. Otra de las normas internacionales a los cuáles Ecuador se ha acogido con respecto al RSE y que se vincula al área de la producción agrícola, son las Normas GLOBALG.A.P, que nace de la organización sin fin de lucro que lleva su mismo nombre y que es la encargada de recopilar las certificaciones de buenas prácticas agrícolas para todas las cadenas de comercialización (Sánchez, 2016). 
Según lo manifestado por el gerente de Global Forum en Ecuador, más de 970 empresas ecuatorianas cuentan con el certificado de Global Gap. Para tener acceso a esta certificación, las empresas deben aprobar criterios de inocuidad alimentaria, trazabilidad, aseguramiento de la calidad, seguridad ocupacional, buen manejo de productos de uso agrícola y que resultan en su mayoría nocivos para el ser humano y por ende para el entorno social. La obtención de esta certificación puede demorar más de un año, dependiendo si la empresa es pequeña o grande (Anchundia \& Quinatoa, 2018).

La certificación, además, facilita el ingreso de los productores a los procesos de exportación contribuyendo a la expansión del mercado. En la actualidad Global Gap se ha establecido en el mercado global como requisito indispensable para en cuanto a las buenas prácticas agrícolas se refiere, logrando que los derechos del consumidor se vean reflejados en la producción agrícola de mas países. En este sentido, Ecuador al ser uno de los máximos exportadores de banano en el mundo, es el país con mayor superficie de banano certificada por Global Gap, seguido por Colombia, Costa Rica y la República Dominicana.

El marco legal en que se basa la RSE, es la regulación de los organismos externos, la propia normativa nacional, como, por ejemplo, la aplicada por la Superintendencia de Economía Popular y Solidaria del Ecuador, así como, los propios estamentos de cada empresa. Como normativa principal en el RSE está El Pacto Global, iniciativa de la ONU que quiso establecer en los procesos de producción, el acatamiento a diez principios básicos, principios basados en cuatro ejes: los derechos humanos, situaciones laborales, cuidado de la naturaleza y procesos libres de especificar.

En los derechos humanos, invita a las empresas a reconocer y respetar todos los derechos humanos reconocidos universalmente. Considerar el tema de los derechos humanos enmarcado al área empresarial no es algo nuevo, este tema fue una de las bases que sustentó el nacimiento de las Naciones Unidas, organismo creado para garantizar la paz universal y el desarrollo de las naciones (Gómez \& Riquelme, 2019).

Los derechos laborales se hacen hincapié a la libertad de asociación, la eliminación de trabajo forzoso, la erradicación del trabajo infantil y toda práctica discriminatoria en el trabajo. Pues, por mucho tiempo se creía que el tema del respeto de los derechos humanos eran un tema que sólo debía preocupar al Estado, y que el papel de la empresa era tan solo de asumir un deber general de cumplimiento de tales derechos, sin que las empresas tuvieran un papel importante en la promoción y defensa (Bonilla, 2017).

En relación con la protección del medio ambiente, se insta a la prevención en el daño a la naturaleza, la responsabilidad ambiental y el crecimiento tecnológico respetuoso del medio ambiente. Y, el último eje que aborda el Pacto Global es el combate a todas las formas de corrupción, incluidos el soborno y la extorsión. 
Los principios del Global Compact, es un código de referencia a nivel mundial, esta normativa constituye una iniciativa voluntaria para aplicar diez principios universales en materia de RSE basados en los ejes de: derechos humanos, derechos laborales, medio ambiente y corrupción.

Otra de las normativas que han sido referentes para las empresas que buscan adaptarse a la RSE son las normativas de la familia ISO. Las normativas de Organización Internacional de Normalización (ISO), es aquella estructura sistemática de alcance mundial y está integrada por 162 países, incluido Ecuador. Es una organización de naturaleza no gubernamental. La misma busca promover el desarrollo de la estandarización y las actividades relacionadas con ella en todo el mundo.

La mayoría de las normas internacionales adoptan un enfoque basado en procesos, cuando se desarrolla, implanta y mejora la eficacia de un Sistema de Gestión de la Calidad (SGC), con el objeto de aumentar la satisfacción del cliente. Un enfoque basado en procesos, dentro de un SGC, destaca la importancia de la comprensión y el cumplimiento de los requisitos, la necesidad de considerar los procesos en términos que aporten valor, la obtención de resultados del desempeño y eficacia del proceso, y la mejora continua de los procesos basada en mediciones objetivas. En las organizaciones de hoy, la dirección debe tomar decisiones a tiempo basándose en hechos. La implantación y certificación de un SGC, basado en esta norma, proporciona una serie de herramientas para priorizar la toma de decisiones, como son la definición de indicadores claves y sus objetivos, así como la identificación de acciones a corregir o mejorar en todas las áreas de la organización (ISO 9001 Gestión de la calidad ).

Otras normativas, dentro de las cuales tenemos la ISO 14001, encargada del Sistema de Gestión Ambiental; la ISO 14004 que se basa en los principios de un Sistema de Gestión Ambiental; la ISO 14010 establece todos los principios generales sobre las auditorias de la Responsabilidad Social dentro de la empresa, es una iniciativa de la escuela europea de excelencia sobre la gestión ambiental; la ISO 19011 sustitutiva de la ISO 14011 abarca una amplia gama de funciones relacionadas con las auditorías en general; la ISO 14012, establece los criterios fundamentales para poder calificar a los auditores; la ISO 14013 establece una guía de consultas a la hora de realizar la revisión de la certificación ambiental. Como vemos, es amplia la familia de las ISO, también se destacan dentro de la temática que nos competen, las ISO 14014, 14015, 14031, 14032, 14060.

Dentro de los integrantes de la familia ISO merece especial consideración la ISO 26000, siendo esta una guía sobre la aplicación de mejores prácticas en responsabilidad social empresarial en todo el mundo. Esta normativa permite la comprensión y la implementación de la RSE mediante el desarrollo de un consenso internacional sobre lo que significa RSE y la difusión de las mejores prácticas realizadas y la asesoría para que se sigan presentando en otras empresas. Al contrario, con otras normas ISO, la ISO 26000 no es una norma de sistema de gestión y por 
lo tanto no es certificable. Esta se presenta como una guía voluntaria que no propone requisitos, sino más bien, pretende ser una guía práctica sobre la operatividad de los procesos relacionados con la RSE.

Sin embargo, la norma ISO 26000 nos aporta siete principios básicos, a través de los cuales se orienta específicamente la gestión del medio ambiente en la empresa, desde el punto de vista de la organización, la gobernanza, los derechos humanos, las prácticas laborales, el medio ambiente, las prácticas de operación, los consumidores y el desarrollo de la comunidad.

Otra de las normativas alineadas a la RSE, es el Reglamento EMAS (Programa Europeo del Ecogestión y Ecoauditoría), que es un sistema voluntario de auditoría de la gestión medioambiental en las organizaciones. Los EMAS se implementan para evaluar y mejorar las acciones de RSE de las organizaciones, para difundir la información pertinente con la gestión ambiental y para mantener un proceso de buena gestión de RS en las empresas. El objetivo específico del Reglamento EMAS es promover la mejora continua del comportamiento medioambiental de las organizaciones.

Siguiendo con el análisis de las normativas internacionales alineadas a la RSE está la SA 8000 (estándar de certificación internacional), la misma que tiene como objetivo, velar por mejores condiciones laborales, mediante el cumplimiento de los acuerdos internacionales y el marco regulatorio de los derechos de los trabajadores y justicia social. También, a más de la normativa anterior están los Indicadores Ethos de RSE, las cuales buscan ofrecer a las empresas un insumo para reforzar su compromiso con la RS y el desarrollo sustentable; esta organización se basa en siete aspectos: valores, transparencia y gobernanza, público interno, medio ambiente, proveedores, consumidores y clientes, comunidad y gobierno y por último sociedad. Además, se considera relevante la normativa AA1000, normativa creada por el Instituto para la Responsabilidad Social y Ética- ISEA (Instituto for Social and Ethical Accountability); esta normativa es un estándar para medir e informar sobre el comportamiento ético en los negocios. El propósito de esta organización británica es, promover el rendimiento de las cuentas a través de una valoración de la empresa, en el marco del cumplimiento de sus expectativas y derechos de los grupos de interés.

Se evidencia que, existen diversas normativas alineadas a la Responsabilidad Social Empresarial; en el siglo actual ha sido evidente el aceleramiento en la búsqueda de la aplicación de la RSE en las empresas. Una de las fuentes principales se encuentra, los acuerdos internacionales, que han colaborado en su expansión y aplicación. También dentro de esta misma lógica, las empresas utilizan ciertas pautas de medición y de esta forma alcanzar su crecimiento.

Una organización muy importante en las políticas de implementación y apertura de responsabilidad social corporativa es el Pacto Mundial de las Naciones Unidas, nace de una iniciativa voluntaria para promover el desarrollo sustentable y la ciudadanía corporativa, el mismo que cuenta con el apoyo de cinco agencias de las 
Naciones Unidas: Programa de las Naciones Unidas para el desarrollo, la Oficina de Alto Comisionado para los Derechos Humanos, la Organización Internacional del Trabajo, el Programa de las Naciones Unidas para el Desarrollo Industrial y el Programa de las Naciones Unidas para el Medio Ambiente (Montoya \& Martínez, 2012)

\section{Relación de la RSE y el ordenamiento jurídico ecuatoriano}

Como norma general o inicial la RSE surge como una cuestión voluntaria, no imperativa, por lo tanto, cabe preguntarse si su cumplimiento debe exigirse o no. Sin embargo, la misma sociedad tanto nacional e internacional clama por su regulación. Este tema reviste de importancia en los dos sectores: por un lado, el empresarial, que prefiere que la RSE siga siendo voluntaria, y, por otro lado, la sociedad civil y las ONG que desean que al menos algunas normativas sean jurídicamente exigibles.

En este orden de ideas, el tema de la RSE aplicable especialmente al área laboral y de los derechos humanos, cobra importancia en los últimos tiempos, sobre todo porque estas áreas son de gran interés a nivel internacional. Existen postulados normativos que exigen el cumplimiento mínimo de sus enunciados. Dentro de estos instrumentos normativos están: La Declaración Universal de Derechos Humanos, La Organización Internacional del Trabajo, El Convenio 87 sobre la Libertad Sindical y la Protección del Derecho de Sindicación, El Convenio 29 sobre el Trabajo Forzoso, El Convenio 111 sobre la Discriminación en el empleo (Camacho, 2015).

Al decir de (Kun, 2015) el derecho del Trabajo y la RSE van de la mano, a pesar de tener diferentes instrumentos de regulación, persiguen objetivos similares, siendo uno de ellos el equilibrio entre la eficiencia del negocio y la protección social, desarrollar medidas que garanticen un mejor trato a los trabajadores, de una manera socialmente responsable, modificando las culturas internas de las mismas. La RSE crea una nueva visión en las empresas, resaltando nuevos enfoques éticos y normativos; visión que es acogida por grandes empresas ecuatorianas, que además adoptan seguir las normativas legales vigentes.

Los lineamientos internacionales de la RSE han servido de sustento y guía en la aplicación de este modelo en Ecuador, sobre todo en aquellas empresas que desean ser responsables y sostenibles. Un papel muy importante lo han tenido los diversos organismos internacionales, la Organización Internacional del trabajo (OIT), la CEPAL, la International Organization for Standardization (ISO), entre otros. La norma ISO 26000 coadyuva para que las empresas demuestren su compromiso con la RSE, encaminados sobre todo a los derechos laborales, cuidados del medio ambiente, protección a los consumidores y usuarios y desarrollo social (Remache, Villacis, \& Guayta, 2018).

Merece especial referencia las normas ISO 26000, ya que éstas han sido una guía de orientación para las empresas, organizaciones, universidades, entidades del 
Estado, entre otros actores. Esta normativa tiene como visión plasmarse como un referente a nivel mundial que busca entre otros la sostenibilidad ambiental y el desarrollo sostenible (Andía, 2015).

La relación existente entre la RSE y el ordenamiento jurídico ecuatoriano es evidente, ya que muchas de las leyes ecuatorianas son concordantes con normativas internacionales no obligatorias y también con las obligatorias, como por ejemplo con lo regulado en los Tratados y Convenios Internacionales, que son normativas vinculantes para el país. La RSE trata de garantizar una convivencia armónica en varios sectores: laborales, ambientales, sociales, que es lo que persigue un ordenamiento jurídico de determinado país.

\section{La Responsabilidad Social en el sector bananero}

El sector bananero se caracteriza por ser uno de los sectores productivos más importantes de país, pese a las continuas crisis que le ha sobrevenido, se mantiene en una posición referencial, como generador de divisas en el país y como fuente de trabajo para miles de ecuatorianos. Según (Sánchez, 2016) "La exportación bananera representa también el 2\% del PIB general, 26\% del PIB agrícola, 8\% de las exportaciones generales, $27 \%$ de las exportaciones agropecuarias y $20 \%$ de las exportaciones no petroleras".

La forma como se lleva a cabo el proceso de monocultivo del banano es una de las características más problemáticas del sector, pues el uso de químicos altamente peligrosos desencadena una serie de enfermedades a los trabajadores; enfermedades que van desde respiratorias hasta catastróficas como el cáncer. Esto debido a la falta de cuidados y políticas de seguridad empleados para el trabajo.

Otra de las problemáticas identificadas en el área laboral dentro del sector bananero es, relacionado a la inexistencia de contratos laborales y pago de salario por debajo de los establecidos en las tablas sectoriales de cada año. Extensión de horarios por más de 12 horas en los tiempos de cosecha y la polivalencia de las tareas del trabajador (Vitali, 2017).También se ha identificado en el sector bananero, la discrimación del trabajo realizado por mujeres, a pesar de que realizan similar trabajo que un hombre, no existe el mismo reconocimiento salarial, a esto se suma, los despidos por situaciones de embarazos, quedando indefensas en un momento muy importante de sus vidas.

En Ecuador se han realizados importantes cambios en la legislación; en especial en lo laboral y empresarial. Sin embargo, las empresas de manera general no prestan mucha atención a los temas sociales y ambientales, siendo escasas las empresas que de manera voluntaria implementan la filosofía del RSE de manera sostenible (Coba, Díaz, Zurita, \& Proaño, 2017).

Se ha evidenciado en una de las mayores provincias productoras de banano como es la provincia de El Oro que, los empresarios están más interesados del 
proceso comercial del banano y que este le genere ganancias, despreocupándose de la RSE, por lo que se debería prestar mayor atención y ejecutar mejores estrategias para implementar normativas ISO 26000, indicadores de medición continua de RS, así como el uso de manuales de buenas prácticas empresariales (Vera, 2018).

El sector bananero del país, es uno de los sectores más contaminantes, porque en el proceso de cultivo hasta su exportación, las bananeras son focos de alta contaminación por la utilización de productos químicos que no solo afectan a los trabajadores del lugar, sino también a las zonas aledañas. Los plásticos que resultan del proceso, no son tratados adecuadamente, e incluso no son recolectados como basura, los mismos que desembocan en los ríos y mares. Las fumigaciones también es otro aspecto dañino que se debe considerar en las bananeras. Por lo que es importante emprender acciones de auditoría ambiental, capacitación para la correcta manipulación y educación continua.

\section{Exigencia o voluntariedad en la aplicación de la RSE}

La RSE y su aplicación en el ámbito empresarial surge de factores voluntarios, sin embargo, en diferentes países del mundo, se han plasmado diferentes normativas en torno a esta temática. Y, Ecuador no se ha quedado rezagado en esta regulación. Cuando nos referimos al carácter voluntario de la aplicación de los instrumentos de la RSE estamos frente al régimen de softlaw, un ejemplo claro de este régimen son los códigos de buen gobierno en donde se expresa la voluntad y la disposición de la empresa para el reconocimiento de todos los intereses en juego (González \& García, 2006).

El softlaw tiene su importancia en la medida que no es exigible para ninguna empresa, y no está revestido del poder sancionador del Estado. Más bien, se trata de un recurso que es acogido y respetado de manera voluntaria por parte de los involucrados, como parte de un compromiso a respetar. Es decir, es una autorregulación que desean afrontar las empresas. Algunos de los defensores de este sistema, consideran que existe mayor satisfacción en la satisfacción de los consumidores y clientes y que, por lo tanto, no es necesario que exista la exigibilidad de la ley para tener una empresa socialmente responsable (Ibarra, 2014). Ejemplo de este sistema son los instrumentos internacionales que la RSE quiere establecer de manera voluntaria, quedando a discreción de las empresas el acogerlas, careciendo de fuerza obligatoria y su incumplimiento no genera sanción ni afectación en la empresa (Acevedo, Zárate, \& Garzón, 2013).

Lo contrario a este sistema voluntario es el sistema hard law, los defensores de este sistema enfatizan que la RSE no puede dejarse al libre albedrío de las empresas, ya que algunas empresas consideran que la "RSE solo tiene sentido si el costo de desplegar un comportamiento más virtuoso continúa siendo menor que los beneficios” (Vogel, 2005). 
Bajo estos criterios, algunos países han optado por incentivar a aquellas empresas que son socialmente responsables, manteniendo una serie de incentivos para aquellas empresas que cumplan con los estándares de RSE, pudiendo ser, rebajas en los impuestos, destacar y aplaudir su gestión públicamente,

La puesta en marcha de la RSE debe ser considerada como un acto voluntario por parte de las empresas, donde impere la consciencia social, mas no, como una imposición al que se deba ajustar. El sector empresarial a más de obtener un beneficio financiero, busca ser bien visto ante la sociedad, porque eso realzará su nivel competitivo, pues, aquellas empresas que son socialmente responsables, son mayormente reconocidas por el consumidor, y esto conlleva a su divulgación, provocando beneficios en la publicidad de la empresa (Lizarzaburu \& Brito , 2016) . Sin embargo, lo que en la actualidad mueve en gran medida al empresario a acogerse a la RSE es tener armonía con la protección a la naturaleza y por ende al entorno social. En este sentido, hoy en día una empresa no puede estar alejada de las exigencias actuales, siendo estas: creatividad, innovación, mejora de la cadena de producción, calidad y sostenibilidad, aceptación de referentes y normativas internacionales, entre otros. En una investigación profunda realizada en Europa, se pudo verificar que, por ejemplo, en la mayoría de las empresas europeas no conciben la RS como una nueva forma de hacer negocios, sino como una novedosa herramienta de gestión que les permite realizar acciones responsables y comunicarlas (Gil, 2018).

Pues, la alarmante situación que enfrenta el planeta, ha llevado a plantearnos alguna solución para mitigar los daños causados por el hombre. Las consecuencias del calentamiento global se hacen visibles en nuestros días, los desastres naturales se han desencadenado a nivel mundial con efectos desastrosos. La extinción de animales y plantas también nos hacen pensar en una solución. El crecimiento de enfermedades raras y catastróficas también son efectos del mal manejo del medio ambiente. Todos estos ejemplos enunciados son una clara muestra de la urgente necesidad de tener consciencia en la actividad empresarial. Además, tenemos las enfermedades profesionales, y sobre esto, existe la percepción de que las innovaciones no son necesarias, razón por la cual, se siguen utilizando prácticas antiguas que van en desmedro de la salud y condiciones físicas de los trabajadores (Murillo, 2018).

Sin embargo, pese a esta difícil situación, podemos también evidenciar que muchos empresarios, llámense pequeños o grandes, no tienen interés en la RSE, sus actuaciones en desmedro de la propiedad privada, inmoralidad, corrupción, afectación a clientes, violación de derechos, entre otros, no tienen límites, lo importante son sus estados financieros (Moya, 2014).

Las organizaciones deben tener claro que es lo que desean con la RSE, si desean ser realmente responsables con la sociedad, y hacer del RSE parte de su estructura organizacional; ser empresas sólo con programas de responsabilidad social, aplican- 
do únicamente uno de los objetivos del RSE; o simplemente negarse a una realidad empresarial que cada vez toma más vigencia en mundo, debido a la globalización (VV.AA, 2017).

La aplicación de la RSE en los diferentes sectores no es una tarea tan sencilla, es una cuestión de tiempo. Las empresas deberían contribuir a la formación y capacitación de los jóvenes y futuros empresarios, con programas de divulgación de los conceptos empresariales, de emprendimiento, de buena gobernanza y responsabilidad social, así como de transparencia y ética en los negocios (Olcese, 2015) y de esta forma estamos creando una conciencia de responsabilidad social a los futuros empresarios de la sociedad. También es importante que desde la academia debe reforzarse el tema de adhesión al RSE ya que permite alcanzar sostenibilidad y sustentabilidad (Luzurriaga, 2018).

\section{Conclusiones}

Las normativas analizadas tienen su relevancia en la RSE porque su aplicación ayuda a la medición de los estándares necesarios para el logro de objetivos a alcanzar, basados en los diferentes organismos internacionales; los cuales permiten definir si una empresa u organización es socialmente responsable, ya que esta va en sintonía con los derechos humanos, los derechos ambientales y los derechos de los trabajadores.

El desarrollo y evolución de los principios del RSE ha sido significante a nivel mundial, y que en Ecuador estas bases han sido acogidas en muchas empresas, sobre todo, en las multinacionales. Sin embargo, existen otras empresas que no muestran interés en ser socialmente responsables, siendo el sector bananero uno de los sectores que más perjuicio causa al medio ambiente y al sector laboral.

El impacto que llegaría a tener el sector bananero con la aplicación del RSE fuera relevante, pues evitaría causar tanto daño al medio ambiente, a la salud de los trabajadores que ahí laboran y a la sociedad misma, que recibe sus efectos. Por ello, la mejora de nuestras acciones es cosa de nosotros mismos, la empresa busca cada día ser competitiva; todos los fallos, errores, las imperfecciones, los accidentes son sin duda inevitables, son consecuencias de una mala gestión (Fernández, 2018). Sin embargo, la decisión de querer ser una empresa socialmente responsable, le corresponde a los integrantes de cada empresa.

Cuando se hace la afirmación de daños al medio ambiente, se debería indicar que tipo de daños, extensión del daño y si es causa de la aplicación incorrecta de prácticas de RSE. Igual para los trabajadores. Cuantos trabajadores tiene el sector bananero ecuatoriano? Y como es el antes y después de la aplicación de los principios de RSE. 
La adopción de normativas de RSE en el ordenamiento jurídico ecuatoriano es relevante, la puesta en marcha de varios Tratados y Convenios Internacionales ayuda de una $u$ otra forma adecuar la normativa interna a los beneficios que procura una actuación social responsable. En Ecuador aún falta normar de manera específica la RSE en las empresas bananeros, existen adelantos, pero estos no son significativos. Se tiene que trabajar arduamente en el tema laboral, la salud, impacto ambiental y seguridad. Para ello, es indispensable legislar al respecto, capacitación continua de las fortalezas y oportunidades de la RSE en el sector bananero, que permitan cristalizar políticas sociales y medio aplicables a este sector. 


\section{Referencias}

Acevedo Guerrero , J., Zárate Rueda , R., \& Garzón Ruiz , W. (diciembre de 2013). Estatus jurídico de la responsabilidad social empresarial (RSE) en Colombia. $22(2), 303-332$.

Acosta-Véliz, Marjorie, Lovato-Torres, Sofía, \&Buñay-Cantos, Jahaira. (2018). La responsabilidad social corporativa y su rol en las empresas ecuatorianas. Revista Lasallista de Investigación, 15(2), 105- 117. https://dx.doi.org/10.22507/rli. v15n 2 a 8

Anchundia Gende, S., Quinatoa Vera, P., (2018). Análisis de los factores de responsabilidad social empresarial que inciden en las productoras y exportadoras de mango en el cantón Guayaquil. Tesis. Recuperado de http://repositorio.ug.edu. ec/handle/redug/30318

Andía Valencia, W. (julio-diciembre de 2015). La responsabilidad social: análisis del enfoque de ISO 26000. Industrial Data, 18(2), 55-60. doi:1560-9146

Bonilla Sanabria , F. (2017). Comentarios sobre la responsabilidad social empresarial,el derecho societario y la empresa de grupo. (F. Bonilla Sanabria , Trad.) 134Vniversitas , 21-58. Recuperado el 15 junio de 2019, de http://dx.doi. org/10.11144/Javeriana.vj134.crse

Camacho Solís, J. (enero-junio de 2015). Las normas de responsabilidad social. Su dimensión en el ámbito.

LABORAL DE LAS EMPRESAS. Latinoamericana de Derecho Social(20), 3-29. Obtenido de http://biblio.juridicas.unam.mx

Coba Molina, E., Díaz Córdova, J., Zurita Meza, E., \& Proaño López, P. (2017). La responsabilidad social empresarial en las empresas del Ecuador. Un test de relación con la imagen corporativa y desempeño financiero. Ingeniería Industrial. Actualidad y Nuevas Tendencias, 5(18), 23-44. Recuperado el 15 junio de 2019, de https://www.redalyc.org/pdf/2150/215052403003.pdf

Cordero, J. A. (2017). La responsabilidad social empresarial. Una óptica desde los stakeholders. Revista Economía y Política, (26), 103-123.

Chumaceiro, A., Hernández, J. Y., \& Ziritt, G. (2013). Responsabilidad social empresarial y políticas públicas. Revista de Ciencias Sociales (Ve), 19(2), 309-321.

Obtenido de https://www.redalyc.org/articulo.oa?id=28026992010

Fernández, \& Lluch . (2015). Familias empresarias y grandes empresas familiares en america latina y españa:Una visión de largo plazo. BBVA. Recuperado el junio de 2019, de https://ebookcentral.proquest.com

Fernández, G. (2018). La responsabilidad social corporativa como modelo de gestion empresarial. Recuperado el 16 junio de 2019, de https://ebookcentral.proquest.com 
Gil Sánchez. (2018). Responsabilidad social corporativa:Revision crítica de una nación empresarial. Recuperado el 16 junio de 2019, de https://ebookcentral. proquest.com

Gómez, \& Fernández. (2019). La acción social empresarial como instrumento de Justicia Social: la empresa como garante de los Derechos Humanos. Obtenido de https://dialnet-unirioja es.accedys.udc.es/servlet/articulo?codigo $=6816986$

Atehortúa Hurtado, F., (2008). Responsabilidad social empresarial: entre la ética discursiva y la Racionalidad técnica. Revista Escuela de Administración de Negocios, (62), undefined-undefined. 2019]. ISSN: 0120-8160. Recuperado de: https://www.redalyc.org/articulo.oa?id=206/20611457009

Kun, A. (2015). Innovadores métodos de regulación en la periferia del Derecho del Trabajo: propuestas desde la responsabilidad social empresarial, softlaw, Derecho de la Empresa, entre otros. Ius et Praxis,, 21(1), 577-598. Recuperado el junio de 2019, de http://www.redalyc.org/articulo.oa

Lizarzaburu, E., \& Brito , J. (2016). Responsabilidad Social Corporativa y Reputacioón Corporativa en el sector financiero de paises en desarrollo . Journal Globalization, Competitiveness Y Governability, 10(1), 42-65. doi:10.3232

Luzuriaga, K. (2018). Análisis de la evolución de la Responsabilidad Social en el Ecuador, según las corrientes teóricas de Garriga y Melé. Obtenido de: https:// dialnet-unirioja-es.accedys.udc.es/servlet/articulo? codigo $=6850179$

Martínez Herrera, H. (2010). Responsabilidad social y ética empresarial. Ecoe Ediciones.

Montoya Arévalo, B. E., \& Martínez Ramos, P. (2012). Responsabilidad social empresarial: Una respuesta ética ante los desafíos globales. 84. Recuperado el 15 junio de 2019. https://www.kas.de/c/document_library/get_file?uuid=0ade669e-4a46-3edf-bd16-e7eb6403e0fb\&groupld=252038

Morán, C., Rodríguez, L., Torres, M., Aguilar , A., \& Villalta, M. (2016). Stakeholders, responsabilidad social en ecuador Stakeholders, social responsibility in ecuador. Revista Científica Y Tecnológica UPSE, 3(2), 21-30. https://doi. org/10.26423/rctu.v3i2.147

Moreno, M. (30 de Marzo de 2013). RSE en Latinoamérica. ¿Qué es y hacia dónde vamos? Obtenido de EOI: https://www.eoi.es/blogs/ embacon/2013/03/30/rse-en-latinoamerica-\%C2\%BFque-es-y-hacia-donde-vamos/

Murillo Barrera, C. (2018). Evaluación de las condiciones de ambiente de trabajo y empleo como parte de la Responsabilidad Social Empresarial en las empresas de cultivo de banano del cantón Milagro. 118. Recuperado el 15 junio de 2019, de http://repositorio.ucsg.edu.ec/bitstream/3317/11159/1/T-UCSGPOS- MAE-192.pdf 
Olcese, A. (2015). La responsabilidad social, motor del cambio empresarial: Una propuesta española para Europa y América latina. Recuperado el junio de 2019, de https://ebookcentral.proquest.com

Poveda , G., \& Parrales , J. (2018). Una visión a la responsabilidad Social ambiental en el Ecuador. Revista DELOS Desarrollo LocalSostenible, $31 . \quad$ Obtenido de https://www.eumed.net/rev/delos/31/guido-poveda4.html

Remache Rubio, M. M., Villacis Torres, S., \& Guayta Toapanta, N. A. (1 de enero de 2018). La responsabilidad social empresarial vista desde un enfoque teórico. Dominio de las ciencias, 4(1), 550-568. Recuperado el 18 junio de 2019, de http://dominiodelasciencias.com/ojs/index.php/es/index

Sánchez Gutiérrez, J. (2016). Las normas de calidad GLOBAL G.A.P. y su incidencia en la responsabilidad social empresarial - Caso Bananera Pacidel S.A. del cantón El Guabo, provincia de El Oro. Maestría , Universidad Andina Simón Bolívar, Quito . Recuperado el 15 junio de 2019, de http://repositorio.uasb.edu. ec/bitstream/10644/5565/1/T2251 - MBA-Sanchez-Las\%20normas.pdf

Schulze, F., \& Pszolla, V. (2011). Situación de la responsabilidad social empresarial en Europa. Strategy \& management.

Business review, 2(2), 1-21. Recuperado el 15 junio de 2019, de https://mba.americaeconomia.com/sites/mba.americaeconomia.com/files/responsabilidadsocial. pdf

VV.AA. Responsabilidad social empresarial : Decisiones, reflexiones y casos de estudio. (2017). Retrieved from https://ebookcentral.proquest.com

Vera Salas, L., (2018). Factores que explican la Responsabilidad Social Empresarial en el Sector Exportador de la Provincia de El Oro, Ecuador. Recuperado de http://192.188.52.94:8080/bitstream/3317/10776/1/T-UCSG-POSMAE-164.pdf

Villacís, C., Suárez, Y., \& Güillín, X. (2016). Análisis de la Responsabilidad Social en el Ecuador. Revista Publicando, 3(8), 452-466. Recuperado 20 el junio de 2019. Dialnet-AnalisisDeLaResponsabilidadSocialEnElEcuador-5833426

Vitali, S. (enero-junio de 2017). Precariedad en las condiciones de trabajo y salud de los trabajadores del sector bananero del Ecuador. Salud de los trabajadores , 25(1), 9-22. Recuperado el 20 junio de 2019, de http://www.redalyc.org/ articulo.oa

Wojtarowski, A., Silva, E., Piñar, M., \& Negrete, J. (2016). La Responsabilidad Social Empresarial como pieza clave en la transición hacia el desarrollo sustentable en el sector turístico. PASOS. Revista de Turismo y Patrimonio Cultural, 14(1), 127-139. Obtenido de https://www.redalyc.org/articulo.oa?id=88143642009 
Universidad Técnica de Machala

Ediciones UTMACH

Primera edición en español 2020

PDF interactivo 



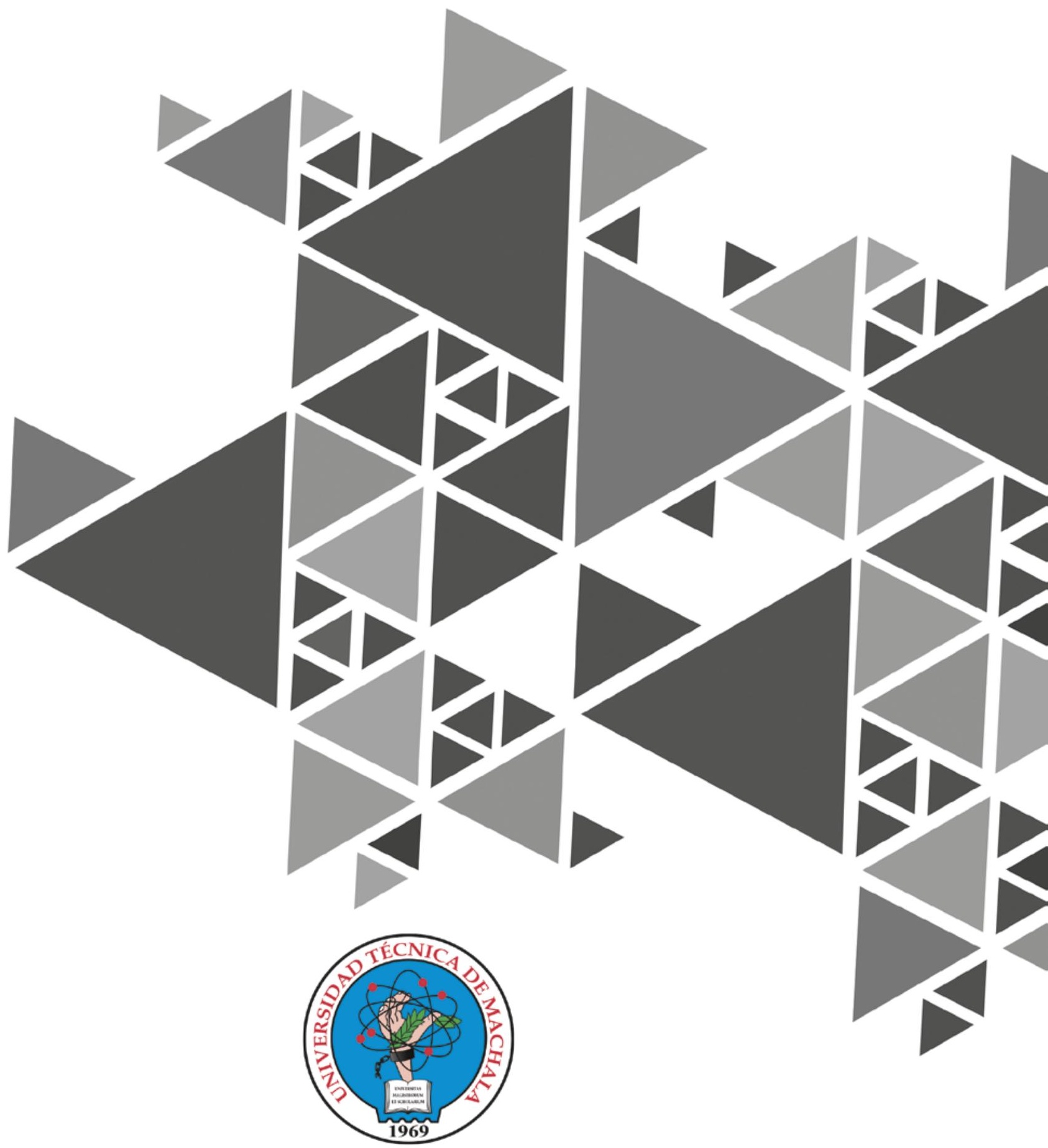

Universidad Técnica de Machala

Dirección de Investigación 